\title{
Programas sociales: transferencias públicas y privadas en México en relación con la inequidad y la pobreza, 2006-2010*
}

José Luis Manzanares Rivera**

\section{RESUMEN}

El objetivo es hacer una valoración de la incidencia de los programas gubernamentales de trasferencias sociales, así como de las transferencias privadas, sobre la distribución del ingreso y la reducción de la pobreza en México para el periodo 2006-2010. La metodología se basa en la estimación de indicadores sobre distribución del ingreso y en herramientas econométricas como la regresión ponderada geográficamente a partir de información de la ENIGH 2006 y 2010. Las conclusiones indican una mejoría en la distribución del ingreso de $2.7 \%$ del índice de Gini durante el lapso estudiado, principalmente para los deciles de menor ingreso, con una tasa de crecimiento equivalente en pobreza (PEGR) de $16 \%$ en 2010. En términos geográficos, se encontró una eficacia limitada del programa Oportunidades, mientras que se observa un impacto positivo de los recursos provenientes de las remesas.

Palabras clave: transferencias públicas, programas sociales, distribución del ingreso, pobreza, inequidad.

Clasificación JEL: D31, I38, I28, H53.

\begin{abstract}
The goal of the paper is to evaluate the incidence of public and private transfers and their contribution to income distribution and poverty reduction in Mexico during 2006-2010. The methodology is based on estimation of distributional measures and econometric tools such as a geographically weighted regression using data from the ENIGH (National Survey of Household Income and Expenditures) 2006 and 2010. The conclusions indicate an improvement on income distribution associated to transfer payments equivalent to $2.7 \%$ of Gini Index over the period 2006-2010, mainly improving the distribution of lower income deciles, with an estimated poverty equivalent growth rate of $16 \%$ for year 2010. From a geographic perspective a limited efficiency was found for the public transfer program Oportunidades, while a rather positive impact, although marginal, was found for private transfers via remittances.
\end{abstract}

Keywords: public trasfers, social programs, income distribution, poverty, inequality. JEL classification: D31, I38, I28, H53.

* Fecha de recepción: 11/07/2012. Fecha de aceptación: 06/06/2013.

** Profesor-investigador de El Colegio de la Frontera Norte. Correo electrónico: jlmanzanaresrivera@gmail.com. 


\section{INTRODUCCIÓN}

El impacto que los recursos asignados mediante programas sociales tienen sobre la distribución del ingreso y la reducción de la pobreza es un tema que ha cobrado relevancia internacional, en particular en Latinoamérica, donde persisten grandes brechas en esta materia. De este modo, es ampliamente reconocido el efecto negativo de la inequidad del ingreso sobre las condiciones de bienestar de la población (Duclos, 2009) y el argumento generalmente aceptado es que incluso en un marco de crecimiento económico la inequidad implica una reducción más lenta de la pobreza.

En México, el esquema de transferencias sociales implementado en la última década ha sido estudiado recientemente desde una perspectiva de la eficiencia de los programas focalizados, hacia los que aparentemente ha transitado el Estado de bienestar en el país. Con respecto a este enfoque, Arzate (2010) enfatiza la transición de los programas de trasferencias sociales en México de una etapa de bienestar universal durante el modelo de sustitución de importaciones a una etapa de programas fragmentados cuyo paradigma es la focalización: "Después de la crisis económica de 1994-1995 se ha venido construyendo un subsistema de políticas y programas sociales centrado en la lucha contra la pobreza extrema, un sistema que utiliza el mecanismo de focalización como principal recurso conceptual y técnico para implementar y gestionar los programas gubernamentales" (Arzate, 2010, p. 65).

Para Bibi y Duclos (2005), la evaluación de la efectividad de las políticas de transferencias, se puede abordar a partir de sus efectos en la reducción de la pobreza, de forma tal que, al parecer, el debate que subyace en este contexto es entre las nociones de eficiencia y de equidad. Con esta visión, autores como Kakwani y Pernia (2000), Ravallion y Chen (2003) o Kakwani, Khandker y Son (2004) han desarrollado una serie de indicadores que permiten estimar el grado en el cual los programas de transferencias se reflejan en un crecimiento en favor de los pobres (pro-poor).

Por otro lado, en el contexto de los países de la Organización para la Cooperación y el Desarrollo Económicos (OCDE), el estudio de las implicaciones redistributivas ante el crecimiento económico y el nexo con la reducción de la pobreza ha sido realizado por autores como Foster y D'Ercole (2005). El análisis empírico de estos autores asocia una baja efectividad de los esfuerzos para reducir la inequidad y la pobreza a un débil sistema de transferencias públicas, incapaz de alcanzar a los que menos tienen: "Impacts on inequality... have so far 
been muted because of a reduction in the effectiveness of public transfers and taxes in reaching those with greater needs" (Foster y D'Ercole, 2005, p. 3).

Si bien el análisis de los efectos de los programas de transferencias sociales sobre la redistribución de los recursos cobró relevancia mundial desde la década de los noventa (Atkinson, 1997), en México relativamente pocos trabajos han abordado el balance entre el costo de los programas y sus beneficios. Notablemente, el trabajo de Huesca (2010) pone de relieve la necesidad de analizar la eficiencia de estos programas y presenta estimaciones a nivel nacional en el caso del Programa de Desarrollo Humano Oportunidades.

Sin embargo, las diferencias en los niveles de pobreza y la dinámica económica de cada estado justifican la incorporación de métodos de análisis que consideren las relaciones territoriales, ya que se asume que una determinada política social de transferencias podría funcionar adecuadamente para una región del país y resultar, a su vez, ineficiente en otra.

En este sentido, el objetivo de este trabajo es hacer una valoración de la incidencia de los programas gubernamentales de transferencias sociales y de las transferencias privadas en el ingreso de los hogares, respecto a su contribución en la redistribución del ingreso y la reducción de la pobreza en México, entre 2006 y 2010. En particular, se busca mostrar la efectividad a nivel estatal de programas representativos de transferencias según el origen del recurso, público o privado.

La hipótesis de la investigación plantea como premisa que la aplicación de una metodología que desagrega el impacto de las transferencias, tanto públicas como privadas, sobre los niveles de inequidad del ingreso podría aportar elementos para entender la incidencia de estos recursos sobre la reducción de los niveles de pobreza.

Para lograr estos objetivos, el estudio se sustenta en la metodología propuesta por Ravallion y Chen (2003) y Kakwani y Pernia (2000) como herramienta para evaluar el impacto y el carácter en favor de los pobres de los recursos destinados a transferencias, utilizando microdatos de la Encuesta Nacional de Ingresos y Gastos de los Hogares (ENIGH) para los años 2006 y 2010 (INEGI, 2006; 2010).

Adicionalmente, se realiza un ejercicio estadístico usando la técnica econométrica de regresión ponderada geográficamente para mostrar posibles impactos regionales a partir de los microdatos del Módulo de Condiciones Socioeconómicas de la ENIGH 2010 (INEGI, 2010 b). 
Además de la presente introducción, en la sección I se presentan los antecedentes teóricos y empíricos sobre las transferencias sociales y su impacto en la distribución del ingreso y en la atención a la población en pobreza. La sección II se ocupa de la evolución de las transferencias sociales gubernamentales y privadas en el periodo 2006-2010, mientras que en la sección III se despliega un ejercicio estadístico aplicando la técnica de regresión ponderada geográficamente. Finalmente, se analizan los resultados y se presentan las conclusiones.

\section{Programas de transferencias sociales como mecanismo para LOGRAR OBJETIVOS DE REDISTRIBUCIÓN Y REDUCCIÓN DE LA POBREZA}

¿De qué manera puede influir la política pública en las condiciones socioeconómicas de la población? La idea dominante es que el gasto público, a través de programas de trasferencias, ${ }^{1}$ es una herramienta importante para reducir la pobreza tanto en países desarrollados como en aquellos en desarrollo. Desde una perspectiva teórica, autores como Van de Walle (1995) se refieren al esquema de transferencias como un mecanismo para alcanzar objetivos de redistribución; esta corriente parte de la idea de que la inequidad tiene efectos adversos sobre el bienestar, (Rawls, 1971).

Los nuevos planteamientos teóricos abordan la relación inequidad-pobreza-crecimiento y la presentan como un proceso en el que sus elementos son interdependientes. Al respecto, Kakwani Khandker y Son (2004) basan su argumentación en el hecho de que el crecimiento del ingreso está acompañado por cambios en el nivel de inequidad de la población y dado que un incremento en la inequidad reduce los efectos que pudiera tener el crecimiento para alcanzar los objetivos de mitigación de la pobreza, entonces es necesario estimar los efectos redistributivos del crecimiento. Para ello, proponen una serie de indicadores como la poverty equivalent growth rate (PEGR), o tasa de crecimiento equivalente en pobreza, mediante la cual un nivel determinado de crecimiento en el ingreso puede clasificarse como un programa en favor de los pobres "si el cambio en la inequidad que acompaña el crecimiento reduce el nivel de pobreza total" (Kakwani, Khandker y Son, 2004, p. 10).

\footnotetext{
${ }^{1}$ En Estados Unidos por ejemplo se destinaron 405000 millones de dólares (o $2.7 \%$ del PIB) a programas suplementarios del ingreso durante el año fiscal 2011 (СBO, 2011). Éstos incluyen programas para población en situación de pobreza, mujeres con hijos en situación vulnerable, asistencia a la población discapacitada, etc.
} 
En este contexto, los programas de transferencias sociales, al contribuir al crecimiento del ingreso de los hogares, producen un cambio en la distribución y son susceptibles de evaluación a partir de la reducción en los niveles de pobreza que generan de acuerdo con la definición previa. Una alternativa para la estimación de los efectos del crecimiento en el ingreso en la reducción de la pobreza es presentada por autores como Datt y Ravallion (1992), quienes caracterizan este impacto con base en tres parámetros únicamente: la línea de pobreza, el ingreso promedio de la distribución y la curva de Lorenz que representa la estructura relativa de inequidad en el ingreso. A partir de estos parámetros, los autores descomponen el cambio en el nivel de pobreza en tres componentes: uno atribuible al crecimiento del ingreso, otro asociado a la redistribución generada y un componente residual.

En el terreno del análisis de política pública, esta línea teórica que vincula el crecimiento, la inequidad y la pobreza ha sido retomada por autores como Bourguignon y Spadaro (2006); la particularidad de su propuesta de análisis es que se basa en la técnica de microsimulación como una herramienta de evaluación, que utiliza microdatos provenientes, por ejemplo, de las encuestas nacionales que incluyen características del ingreso y gasto de los hogares. Este enfoque tiene un abordaje ex ante, es decir, permite observar los cambios generados por una política de forma hipotética antes de que en efecto ocurran, lo que resulta ventajoso desde el punto de vista de la selección de programas que optimicen la aplicación de recursos públicos.

En México, este enfoque puede resultar útil ante la orientación de la política social de los últimos años, inserta en un contexto macroeconómico en el que se busca la estabilidad y el crecimiento, pero en el cual el diseño de programas de asistencia ha sido cuestionado desde la perspectiva académica ante la inercia política que aparentemente conllevan estas acciones.

En el ámbito empírico, los indicadores propuestos para descomponer los efectos redistributivos y del crecimiento en el ingreso han sido implementados con datos de países en desarrollo como Brasil e India. Datt y Ravallion (1992) encuentran que durante los ochenta la reducción en la pobreza observada en India se asocia en gran medida a un efecto redistributivo favorable, mientras que en el caso de Brasil ocurrió lo opuesto debido a un deterioro en la distribución del ingreso.

Por parte de la metodología que evalúa el carácter en favor de los pobres de los esfuerzos de política y el crecimiento económico, Kakwani, Khandker y Son (2003) destacan que "The most important goal for the developmental 
effort has become poverty reduction, which can be achieved by economic growth and/or by the distribution of income" (Kakwani, Khandker y Son, 2003, p. 1). Y usando datos de la década de los noventa en países del sureste asiático, como Tailandia, Corea y Vietnam, encuentran que los dos últimos han avanzado en un crecimiento basado en políticas en favor de los pobres, no así el caso de Tailandia. En su análisis, las políticas públicas con programas sociales implementados en el periodo posterior a la crisis asiática de esa década jugaron un papel fundamental al establecer una red de seguridad social complementaria a los mecanismos familiares tradicionales ${ }^{2}$ (Kakwani, Khandker y Son, 2003, p. 20).

En el caso de México, el trabajo de Scott (2005) constituye una primera aproximación al entendimiento de los programas de transferencias que surgieron al inicio del sexenio 2006-2012 -como fue el 70 y Más, creado en 2007 y con antecedentes que se remontan a $2003^{3}$ (Gobierno Federal, 2007, p. 35)- al presentar un análisis del esquema de seguridad social en el contexto de desigualdad y pobreza que pone énfasis en las consecuencias de éstas para los adultos mayores. Con datos de la ENIGH 2002, dicho autor estima que las transferencias por pensiones en México de hecho incrementan la desigualdad ${ }^{4}$ y propone una transición del esquema polarizado vigente a uno de carácter universal.

Esfuerzos más recientes, como el de Huesca (2010), realizan el análisis de Oportunidades en un contexto de debate entre focalización y universalismo de los programas de trasferencias sociales en México. Mediante la metodología de microsimulación, este autor encuentra que el efecto de este programa en la disminución de la pobreza es marginal (Huesca, 2010, p. 195). Para su análisis, se basa en los microdatos de la ENIGH publicados en 2008.

Una crítica fundamental a la metodología de esta vertiente de trabajos es manifestada por autores como Rubalcava (2010), quien argumenta una limitada referencia al plano territorial y a las implicaciones regionales de la aplicación de los programas de transferencias. Al respecto, esta autora subraya: "La estrategia sugerida consiste en explotar intensivamente la información disponible en las

${ }^{2}$ Los casos de Corea y Vietnam resultan ilustrativos de la aplicación de programas de política pública con objetivos de redistribución del ingreso que acompañados de otras medidas orientadas al crecimiento económico han generado una reducción en los indicadores de pobreza.

${ }^{3}$ Los antecedentes de este programa se remontan a 2003 con el Programa de Atención a Adultos Mayores, según lo establecen las propias reglas de operación del 70 y Más de 2007 (SEDESOL, 2012, p. 7).

${ }^{4}$ El autor estima con datos de la ENIGH 2002 que "la relación en el ingreso medio total por persona entre el decil más rico y el más pobre es de 28:1 (el coeficiente de Gini $=0.49$ ) [ ...] si consideramos sólo el ingreso por pensiones esta relación es de 287:1 (Gini = 0.67)" (Scott, 2005, p. 61). 
fuentes oficiales y aprovechar para ello el amplio abanico de instrumentos y técnicas de análisis estadístico y geográfico" (Rubalcava, 2010, p. 229).

En suma, pareciera haber un consenso teórico sobre los efectos redistributivos positivos de la aplicación de programas de transferencias sociales, sin embargo, en términos empíricos persiste la controversia, ya que el resultado respecto a los objetivos de redistribución se asocia al carácter en favor de los pobres y a la modalidad de aplicación de tales programas: de corte universal o focalizado. En cualquier caso, la inclusión de una perspectiva territorial permitiría tener elementos adicionales que contribuyan a un diseño eficiente de estos programas.

Adicionalmente, una ventaja de evaluar la efectividad de estos recursos a partir de microdatos es que permite distinguir entre el carácter progresivo o regresivo de la aplicación de estos recursos. En este sentido, México constituye un caso de estudio de interés, dada la elevada inequidad en la distribución del ingre$\mathrm{so}^{5} \mathrm{y}$ también debido a los esfuerzos que se han realizado por las recientes administraciones para diseñar programas de transferencias sociales con objetivos de reducción de la pobreza.

\section{Transferencias Públicas y PRIVADAS En MÉXICO, INEQUidad Y POBREZA}

\section{Comportamiento de los ingresos por transferencias en 2006 y 2010}

En esta sección se hace una caracterización del comportamiento de los ingresos por transferencias durante el periodo 2006-2010 en México, en particular aquellas de las que se conoce su fuente y se pueden asociar con un programa institucional específico.

Las transferencias se clasifican para efectos de este análisis en gubernamentales y privadas; las primeras son el resultado de la aplicación de programas de política pública con financiamiento federal, estatal o municipal. A su vez, las segundas representan esfuerzos por parte de las propias familias u organismos privados para mitigar las condiciones sociales adversas, como es el caso de las remesas o el otorgamiento de becas particulares.

Los programas representativos en materia de transferencias sociales gubernamentales de la pasada administración federal fueron Oportunidades, Pro-

\footnotetext{
${ }^{5}$ Incluso en el contexto de Latinoamérica y el segundo con mayor inequidad entre los países de la OCDE, sólo por debajo de Chile (OCDE, 2011).
} 
campo, 70 y Más (que se inició en 2007, poco después de arrancado el sexenio del expresidente Felipe Calderón Hinojosa), el Programa de Apoyo Alimentario y, en menor medida, el Programa de Empleo Temporal. Los tres últimos no se consignan hasta la edición 2010 de la ENIGH (INEGI, 2010 a).

De acuerdo con el objetivo 17 del eje 3 "Igualdad de Oportunidades" del Plan Nacional de Desarrollo 2007-2012, los recursos otorgados por dichos programas están orientados a disminuir la marginación y el rezago que enfrentan los grupos sociales vulnerables ${ }^{6}$ (Diario Oficial de la Federación, 2007, p. 84). De este modo, el análisis del impacto logrado puede abordarse mediante sus efectos sobre los indicadores de inequidad y pobreza.

La gráfica 1 muestra los ingresos de los integrantes de los hogares por transferencias según el programa y el tipo de recurso, ya sea gubernamental o privado, con datos de 2010 .

Gráfica 1. Transferencias gubernamentales y privadas según programa. Ingreso per cápita por tipo de localidad (2010)

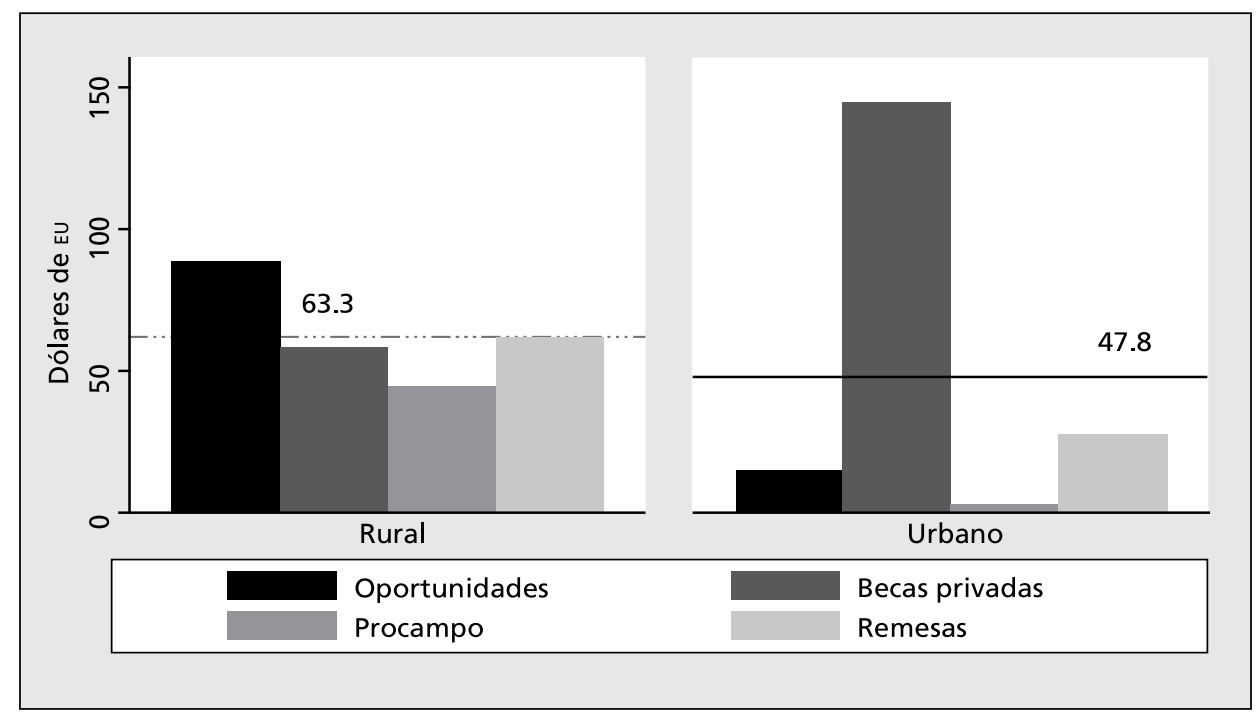

Nota: Ingresos deflactados a agosto de 2010. Localidad rural: menos de 2500 habitantes. Fuente: Elaboración propia con datos de INEGI (2010a). Deflactor del Banxico: IPC.

\footnotetext{
${ }^{6}$ Se estima que uno de los grupos en situación más vulnerable en términos de ingresos es la población de 70 años y más. Este grupo crece en México a un ritmo acelerado, con un incremento de $54 \%$ en la última década, en la que ha pasado de 3.1 millones de personas en 2000 a 4.8 millones en 2012.
} 
Se observa que los ingresos por transferencias son relativamente más elevados en las zonas rurales que en las urbanas, pues en las primeras el ingreso promedio por transferencias (privadas y gubernamentales) en 2010 fue de 63.3 dólares por persona, lo que equivale a unos $3217^{7}$ pesos mensuales por hogar, mientras que en el ámbito urbano (localidades con 2500 o más habitantes) el monto promedio fue de 47.8 dólares por persona, lo que implica una brecha de $32.4 \%$.

Este patrón muestra una aplicación progresiva de los recursos públicos, ya que como se ha observado en términos empíricos, en México existe una mayor incidencia de pobreza en el ámbito rural. Así lo documentan Boltvinik y Damián $(2001)^{8}$ para México, aunque también lo han hecho Kakwani, Khandker y Son (2004) para el entorno internacional.

Si bien en promedio este esquema de transferencias de 2010 favoreció a los hogares rurales, ciertos rubros específicos fueron notablemente mayores en el entorno urbano, por ejemplo, los ingresos por becas privadas, con 144.9 dólares por persona comparados con sólo 58.2 dólares para las áreas rurales. Estas variaciones indican que el impacto de la aplicación de recursos públicos a transferencias no es homogéneo, sin embargo, un análisis a escala estatal de esta relación podría aportar mayor información.

En la ENIGH 2010 (INEGI, 2010 a) se incluyen tres programas adicionales: el programa 70 y Más, ${ }^{9}$ el Programa de Empleo Temporal (PET) y el Programa de Apoyo Alimentario (PAL). ${ }^{10}$ Éstos presentan distintos niveles de focalización; destaca el PET, que se considera de autofocalización, es decir, para que el individuo reciba el apoyo es necesario que acepte participar en proyectos de beneficio comunitario y se aplica en comunidades rurales de hasta 15000 habitantes (Gobierno Federal, 2007, p. 40).

${ }^{7}$ El tamaño promedio del hogar rural en México es de 4.3 integrantes (ligeramente mayor que el hogar urbano típico). El tipo de cambio considerado es el promedio del periodo 2006-2010= 11.82 pesos mexicanos por dólar.

${ }^{8}$ Durante los gobiernos previos a las administraciones del cambio, los niveles mayores de pobreza rural se asocian, como demuestra el autor, a la subestimación de la línea de pobreza.

${ }^{9}$ El programa 70 y Más se implementó a partir de 2007, de acuerdo con la propia Secretaría de Desarrollo Social (SEDESOL, s.f.).

${ }^{10}$ En el cuestionario de 2010, a diferencia del de 2006, se incluyen también los rubros de Beneficios de otros programas sociales y Beneficio de otros programas para adultos mayores, sin embargo, en el presente análisis no se incluyen explícitamente estos recursos ya que no es posible identificar su procedencia y, por lo tanto, no se pueden asociar a una determinada política de transferencias. 
El PAL, por otra parte, es un complemento al programa Oportunidades, que fundamentalmente busca atender a familias con niños menores de cinco años, con la particularidad de que se implementa en poblaciones marginadas donde no se aplica el Oportunidades por falta de acceso a servicios de salud o educación (Diario Oficial de la Federación, 2010, p. 3).

En cuanto al rubro de becas, las transferencias de carácter privado prácticamente triplican los recursos del gobierno destinados al mismo renglón. Así, en 2010 los hogares urbanos que recibieron estos recursos ascendieron a $4.8 \mathrm{mi}-$ llones y promediaron un monto de 1712 pesos mensuales, mientras que la vertiente gubernamental otorgó en promedio sólo 604 pesos por hogar, con poco más de ochocientos mil hogares beneficiados.

Por su parte, Oportunidades, el programa de combate a la pobreza emblemático de las administraciones de los años 2000-2012, destinó notablemente una mayor proporción de recursos que el resto de los programas en el ámbito rural, tendencia que genera cierta controversia, en tanto se asocia a una estrategia de focalización ante un escenario fiscal no favorable para la aplicación de transferencias en un esquema universal. En este sentido, el estudio de Huesca (entre otros) describe con claridad la aplicación de los recursos de este programa con datos a 2008 y concluye que son de baja eficiencia (Huesca, 2010, p.201).

\section{Gráfica 2. Transferencias gubernamentales y privadas según programa Ingresos per cápita por tipo de localidad}

(2006)

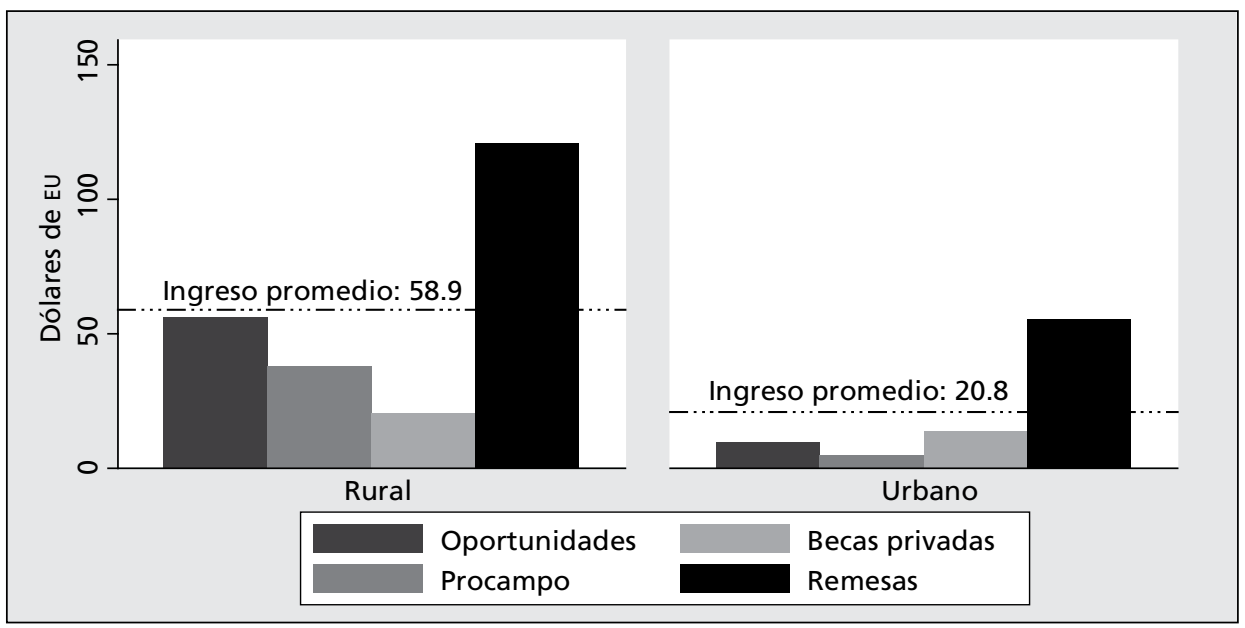

Nota: Ingresos anualizados. Dólares constantes a agosto de 2010. Fuente: Elaboración propia con base en microdatos de INEGI (2006). 
La gráfica 2 muestra la distribución de los recursos por transferencias públicas y privadas, al inicio del sexenio anterior. Es evidente que en el ámbito urbano los ingresos promedio por transferencias registraron un crecimiento en términos reales al pasar de 20.8 dólares en 2006 a 47.2 dólares por persona en el año 2010.

Mientras tanto, en el entorno rural el crecimiento en el mismo periodo fue ínfimo, ${ }^{11}$ al pasar de 58.9 dólares a 63.3 dólares. Los datos comparativos parecen confirmar la corrección parcial del sesgo en la asignación de recursos hacia localidades rurales que autores como Boltvinik y Damián (2001) ya señalaban.

La gráfica 2 también permite apreciar la drástica reducción en el caso de los ingresos por remesas. En el entorno rural, por ejemplo, pasaron de 120.8 dólares por persona en 2006 (o 5568.63 pesos mensuales por hogar) a sólo 62.16 dólares para 2010 (o bien, 2865.48 pesos mensuales por hogar), tendencia que confirma los efectos negativos de la crisis global de 2007-2010.

Tal como ha sido documentado ampliamente desde una perspectiva teórica por Ravallion y Chen (2003), Kakwani y Pernia (2000), Kakwani, Khandker y Son (2003), o bien en aplicaciones empíricas, como la de Duclos y VerdierChouchane (2010), para que las estrategias de crecimiento del ingreso (incluyendo los incrementos generados mediante las transferencias) tengan un impacto efectivo no sólo se requiere que incidan en los niveles de pobreza, sino en la reducción de la inequidad del ingreso entre los individuos. Al respecto, enseguida se analiza la evaluación de los indicadores de pobreza e inequidad en el periodo.

\section{Distribución del ingreso}

La estimación del coeficiente de Gini para el año 2006 es de $0.51,,^{12}$ lo que refleja un entorno de inequidad relevante. La gráfica 3 muestra la concentración del ingreso por deciles, con lo que se tiene una perspectiva de la elevada concentración que el respectivo índice de Gini manifiesta.

Observamos que prácticamente hasta el decil 7, la proporción del ingreso recibido es inferior a $10 \%$ del ingreso per cápita total y, de forma inversa, el último decil de la distribución concentra $39.3 \%$ del ingreso.

\footnotetext{
${ }^{11}$ Con el propósito de validar si esta diferencia es estadísticamente significativa, se llevó a cabo una prueba de hipótesis para diferencias de medias. El resultado indica que se rechaza la hipótesis nula de que la diferencia sea cero entre ambas medias. Los estadísticos de la prueba se presentan en el cuadro A1.

${ }^{12}$ Estimación que considera únicamente el ingreso per cápita antes de transferencias.
} 
70 ECONOMÍA: TEORÍA Y PRÁCTICA • Nueva Época, número 39, julio-diciembre 2013

\section{Gráfica 3. Distribución del ingreso per cápita por deciles} (2006)

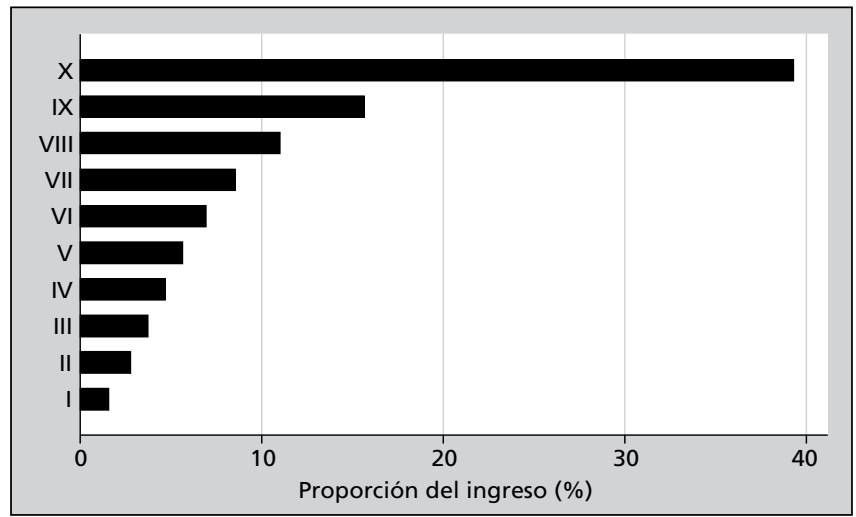

Fuente: Elaboración propia con base en microdatos de INEGI (2006).

Una vez que se incluyen las transferencias tanto gubernamentales como privadas se observa un impacto favorable al generarse una reducción de la desigualdad en la distribución del ingreso de $2.7 \%$ en el índice de Gini al pasar de 0.51 a 0.48 entre el año 2006 y 2010 (véase el cuadro A2 para estimación).

La gráfica 4 muestra las diferencias entre 2006 y 2010 mediante la curva de Lorenz.

Gráfica 4. Curva de Lorenz con la diferencia entre 2006 y 2010

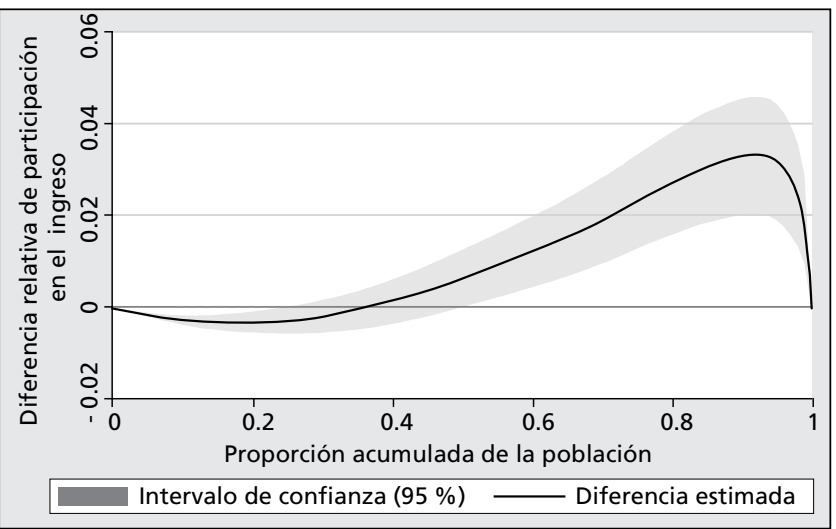

Fuente: Estimación propia con datos de INEGI (2006; 2010). 
Se observa que la mejoría en las condiciones de ingreso se presentó en los deciles de menores recursos, lo que implica nuevamente un efecto progresivo de estas transferencias. Si bien esta mejoría es marginal, concuerda con la relación teórica planteada por autores como Van de Walle (1995) o Kakwani, Khandler y Son (2004) y descrita en la sección I.

No obstante, contrasta con trabajos empíricos sobre México, como el de Scott (2005), quien documenta el incremento de la desigualdad al analizar el caso particular de transferencias por pensiones. Así, la inclusión de una combinación de transferencias privadas y de programas públicos pareciera generar un impacto deseable para los objetivos de redistribución.

\section{Evolución de la pobreza y efecto de las transferencias sobre los índices de crecimiento del ingreso}

La evolución de los niveles de pobreza nos permitirá contar con elementos adicionales para entender el impacto de las transferencias en este periodo. Para este estudio se calcularon dos índices de pobreza: el Foster-Greer-Thorbecke (FGT) y el Sen-Shorrocks-Thon (SST), el primero para representar la incidencia y el segundo porque combina intensidad, incidencia e inequidad. Ambas estimaciones se realizaron para la situación inicial (2006) y final (2010) considerando el ingreso corriente. Adicionalmente, se hizo una desagregación de los ámbitos urbano y rural; en ambos casos se aplicó como umbral de pobreza la línea de bienestar utilizada por el Consejo Nacional de Evaluación de la Política de Desarrollo Social (CONEVAL, s.f.a) para la medición de la pobreza por la dimensión del ingreso.

Cuadro 1. Índice FGT para 2006 y 2010 según tamaño de localidad

\begin{tabular}{|c|l|c|c|c|c|c|}
\hline Tipo & \multicolumn{1}{|c|}{ Tamaño de localidad } & $\begin{array}{c}\text { Índice } \\
2006\end{array}$ & $\begin{array}{c}\text { Línea de } \\
\text { bienestar }\end{array}$ & $\begin{array}{c}\text { Índice } \\
2010\end{array}$ & $\begin{array}{c}\text { Línea de } \\
\text { bienestar }\end{array}$ & $\begin{array}{c}\text { Diferencia } \\
2010-2006\end{array}$ \\
\hline \multirow{3}{*}{ Urbana } & 100000 y más habitantes & 0.27 & & 0.36 & & 0.09 \\
& 15000 a 99999 habitantes & 0.43 & MXN 1732.59 & 0.56 & MXN2 120.04 & 0.13 \\
& 2500 a 14999 habitantes & 0.69 & & 0.68 & & -0.01 \\
\hline Urbana & Total sin desagregar & 0.38 & MXN 1732.59 & 0.45 & MXN2 120.04 & 0.07 \\
\hline Rural & menos de 2500 habitantes & 0.52 & MXN 1 070.57 & 0.64 & MXN 1330.50 & 0.12 \\
\hline
\end{tabular}

Fuente: Elaboración propia con datos de INEGI $(2006 ; 2010)$ y de CONEVAL. 
Al parecer, durante el periodo 2006-2010, los niveles de pobreza se incrementaron. De acuerdo con el índice FGT (cuadro 1$),{ }^{13}$ el incremento en el ámbito rural fue de $12 \%$, al pasar de un índice de $52 \%$ en 2006 a uno de $64 \%$ en 2010. En el entorno urbano el incremento fue de 7\%, es decir que en las ciudades al fin del periodo estudiado el índice era equivalente a 32 millones de personas más en situación de pobreza, 6.9 millones más que en 2006. Este incremento, dada la información sobre inequidad previa, implica que durante el periodo se tuvo un incremento de la cantidad de pobres en el país acompañado de una ligera mejoría en la distribución del ingreso atribuible parcialmente a las transferencias.

Las estimaciones del índice $\mathrm{SST}^{14}$ validan el comportamiento al alza de la pobreza tanto en el ámbito urbano como en localidades rurales. Este indicador para 2006 en el ámbito urbano es de 0.25 y para 2010 de 0.30 . Y para el entorno rural es de 0.34 en 2006 y 0.48 en 2010 (véase el cuadro A3 para estimación).

Para aislar el impacto de las transferencias gubernamentales en el crecimiento del ingreso y mostrar la magnitud en la cual se pueden considerar como una política en favor de los pobres se estimaron los indicadores de Ravallion y Chen (2003), Kakwani y Pernia (2000) y Kakwani, Khandker y Son (2004) usando los datos de 2010 en una situación de ingreso sin transferencias versus una de ingreso con transferencias. El de Kakwani, Khandker y Son también se llama tasa de crecimiento equivalente en pobreza (PEGR, por sus siglas en inglés).

Cuadro 2. Efecto de las transferencias sobre varios índices de crecimiento del ingreso (2010)

\begin{tabular}{lcccc}
\hline Línea de bienestar = MXN 2 120.04 & \multicolumn{4}{c}{ Límite } \\
\hline & Estimado & $\begin{array}{c}\text { Error } \\
\text { estándar }\end{array}$ & $\begin{array}{c}\text { Límite } \\
\text { inferior }\end{array}$ & superior \\
\hline $\begin{array}{l}\text { Tasa de crecimiento (g) } \\
\text { Índices en favor de los pobres }\end{array}$ & 0.15 & 0.00 & 0.14 & 0.16 \\
Ravallion y Chen & 0.21 & 0.00 & 0.20 & 0.22 \\
Ravallion y Chen - g & 0.07 & 0.01 & 0.06 & 0.08 \\
Kakwani y Pernia & 1.07 & 0.08 & 0.92 & 1.22 \\
PEGR & 0.16 & 0.01 & 0.14 & 0.18 \\
PEGR - g & 0.01 & 0.01 & 0.00 & 0.02 \\
\hline
\end{tabular}

Nota: Las estimaciones se realizaron para el entorno urbano. La línea de bienestar es la oficial del CONEVAL (s.f.b) para agosto de 2010.

Fuente: Elaboración propia con datos de INEGI (2010a).

\footnotetext{
${ }^{13}$ Se estimó la versión $\alpha=0$, que muestra la proporción de población que percibe ingresos inferiores a la línea de pobreza.

${ }^{14} \mathrm{El}$ indicador SST $=($ proporción de pobres $) \times($ brecha de pobreza promedio $) \times(1+$ coeficiente de Gini de brecha de pobreza de la población). O bien, $P_{S S T}=P_{0} P_{1}\left(1+G^{P}\right)$.
} 
Como se observa en el cuadro 2, la tasa de crecimiento media del ingreso para el total de la población, una vez que se tiene la aplicación de los recursos por transferencias, es de $15.1 \%$. Sin embargo, el resultado del índice propuesto por Ravallion y Chen (2003), que nos permite estimar la tasa media de crecimiento de los ingresos de la población pobre (aquella con ingresos por debajo de la línea de pobreza establecida), es positivo (21\%), es decir, en términos absolutos el crecimiento del ingreso de la población pobre no sólo es positivo, sino que tiene una magnitud notable, lo que representa un crecimiento en favor de los pobres atribuible al esquema de transferencias aplicado.

De forma similar, la diferencia entre el índice de Ravallion y Chen (2003) y la tasa de crecimiento de los ingresos es positiva, lo que prueba un esquema en favor de los pobres, también en términos relativos. El indicador propuesto por Kakwani y Pernia (2000) es positivo y, dado que la tasa de crecimiento es positiva, es posible afirmar que el impacto redistributivo de las trasferencias es de una reducción absoluta de la pobreza. Adicionalmente, el indicador PEGR es positivo y mayor que la tasa de crecimiento del ingreso, confirmando lo observado por los otros dos indicadores, es decir, que las transferencias tienen un efecto redistributivo en favor de los pobres.

\section{ANÁLISIS ESTADÍSTICO: EL IMPACTO LOCAL}

\section{Aspectos metodológicos}

Con la finalidad de captar el impacto de las transferencias sociales en los niveles observados de pobreza a nivel local, se propone un modelo de regresión espacial. Éste pondera la ecuación de regresión tradicional con los atributos geográficos del área de análisis.

De acuerdo con Xisco, Piccoli y Spadaro (2010), existe un impacto favorable del esquema de transferencias sobre los niveles de pobreza, sin embargo, tal como advierte Scott (2004), es importante distinguir entre el impacto de las transferencias expresamente diseñadas por parte del gobierno para combatir la vulnerabilidad social (que llamaremos transferencias públicas) y el de otros recursos en forma de transferencias privadas que abarcan el esfuerzo por parte de las propias familias o de organizaciones privadas y no gubernamentales para mitigar las condiciones sociales adversas. Por esta razón, en el presente apartado se realiza un contraste entre estos dos tipos de transferencias en tanto su impacto sobre el indicador propuesto de pobreza. 
Para el análisis se utilizan microdatos provenientes del Módulo de Condiciones Socioeconómicas de la ENIGH 2010 (INEGI, 2010 b). Esta encuesta incluye una muestra de 61847 viviendas y permite estimaciones estadísticamente representativas a nivel estatal con un corte urbano y rural. Se asume que la variable que mide indirectamente el nivel de vida es el ingreso corriente de los individuos que se expresó con periodicidad mensual en términos reales al mes de agosto de 2010. Esta variable es usada para el cálculo de pobreza de acuerdo al indicador FGT en la versión simple del índice con el parámetro $\alpha=0$ (proporción de personas con ingreso inferior a una línea de pobreza determinada).

\section{Modelo propuesto}

El objetivo del modelo es analizar el impacto de transferencias públicas y privadas específicas sobre el nivel de pobreza a escala estatal usando la línea de bienestar propuesta por el CONEVAL (s.f.b) para los ámbitos rural y urbano. Ésta expresa el valor de la canasta alimentaria y no alimentaria y equivale a $2120.02^{15}$ pesos en el ámbito urbano y a 1330.50 pesos en el rural a precios de agosto de 2010. Para homologar las estimaciones, las variables independientes del modelo se deflactaron a precios de agosto de 2010. Con esta información se estimó el índice FGT, la variable dependiente del modelo que mide indirectamente la proporción de población que se encuentra en pobreza, según el criterio monetario antes señalado.

Respecto a la selección de las variables independientes para el modelo, la información del Módulo de Condiciones Socioeconómicas de la ENIGH 2010 distingue una amplia variedad de transferencias públicas tales como: 70 y Más, Oportunidades, Procampo, Programa de Empleo Temporal (PET), Programa de Apoyo Alimentario (PAL), y Becas del gobierno, Otros programas para adultos mayores y Otros programas sociales, como categorías genéricas.

Se realizó una fase de exploración en la que se descartaron de ser incluidos en el modelo los siguientes programas y categorías: Procampo y PAL, ya que están orientados fundamentalmente al ámbito rural; PET, debido a que sólo se implementó en un subconjunto de estados; Otros programas sociales y Otros programas para adultos mayores, pues si bien podrían contener información rele-

\footnotetext{
${ }^{15}$ Esta línea de bienestar que incluye una canasta básica más una canasta no alimentaria es una medida comúnmente usada en el análisis de la pobreza en México y su valor se ajusta continuamente para incluir artículos representativos. Por otro lado, el valor reportado de 2120.02 pesos corresponde a precios de agosto de 2010.
} 
vante no es posible conocer el origen de los recursos, lo que dificulta asociar su desempeño con políticas institucionales especificas; 70 y Más, porque tiene un sesgo hacia localidades rurales al ofrecerse únicamente en áreas de menos de treinta mil habitantes, durante el periodo de estudio. ${ }^{16}$

Por lo anterior, se seleccionaron como variables representativas de transferencias públicas para efectos de este trabajo las becas otorgadas por el gobierno y el programa Oportunidades, que tiene como objetivo atender la condición de pobreza extrema. En el caso de las trasferencias privadas, el Módulo de Condiciones Socioeconómicas de la ENIGH 2010 (INEGI, 2010 b) distingue dos tipos de interés para este estudio: becas provenientes de instituciones privadas o de organismos no gubernamentales y transferencias provenientes del exterior mediante remesas.

La especificación propuesta inicialmente es la siguiente:

$$
\text { ifgt }=a_{0}+\beta_{1} \text { oport }+\beta_{2} \text { becaspriv }+\beta_{2} \text { becasgob }+\beta_{4} \text { remesas }+\mu_{1}
$$

donde ifgt representa la medición indirecta del FGT del nivel de pobreza en los hogares realizada a partir de la línea de bienestar propuesta por el CONEVAL (s.f.b) para agosto de 2010; oport, el ingreso mensual promedio por hogar del programa Oportunidades; becaspriv, los ingresos mensuales promedio por hogar por becas privadas; becasgob, los ingresos mensuales promedio por becas gubernamentales, y remesas, los ingresos por remesas. La relación teórica esperada es inversa en todos los casos. Siguiendo la metodología propuesta por Fotheringham, Brunsdon y Charlton (2002) para estimar modelos de regresión ponderada geográficamente, se calculó en primer lugar la especificación propuesta usando la estimación global de mínimos cuadrados ordinarios, lo que permitió determinar la mejor especificación para posteriormente aplicar la técnica local de regresión ponderada geográficamente.

\section{a) Modelo 1}

$$
\text { ifgt }=a_{0}+\beta_{1} \text { oport }+\beta_{2} \text { becaspriv }+\beta_{3} \text { becasgob }+\beta_{4} \text { remesas }+\mu_{1}
$$

Los resultados de la regresión son los siguientes:

\footnotetext{
${ }^{16}$ En la administración actual de Enrique Peña Nieto, las reglas de operación se modificaron para incluir a localidades mayores de treinta mil habitantes.
} 
76 ECONOMÍA: TEORÍA Y PRÁCTICA • Nueva Época, número 39, julio-diciembre 2013

\begin{tabular}{|c|c|c|c|c|c|c|}
\hline$F(4,27)$ & $=$ & 3.99 & $R^{2}$ & $=$ & 0.3716 & \\
\hline Prob. $>F$ & $=$ & 0.0114 & $R^{2}$ ajustada & $=$ & 0.2785 & \\
\hline $\begin{array}{l}\text { Raíz del error } \\
\text { cuadrático } \\
\text { medio }\end{array}$ & $=$ & 11.434 & & & & \\
\hline ifgt & Coeficiente & $\begin{array}{c}\text { Error } \\
\text { estándar }\end{array}$ & $t$ & $P>t$ & \multicolumn{2}{|c|}{$\begin{array}{c}\text { [Intervalo de confianza } \\
\text { de } 95 \% \text { ] }\end{array}$} \\
\hline Oportunidades & 0.0728 & 0.03 & 2.38 & 0.03 & 0.01 & 0.14 \\
\hline Remesas & -0.0050 & 0.00 & -1.92 & 0.07 & -0.01 & 0.00 \\
\hline Becas privadas & -0.0077 & 0.01 & -1.03 & 0.31 & -0.02 & 0.01 \\
\hline $\begin{array}{l}\text { Becas de } \\
\text { gobierno }\end{array}$ & 0.0028 & 0.01 & 0.52 & 0.61 & -0.01 & 0.01 \\
\hline Constante & 21.45 & 22.10 & 0.97 & 0.34 & -23.89 & 66.79 \\
\hline
\end{tabular}

Fuente: Estimación propia con datos de INEGI (2010b).

Se observa que las variables becas de gobierno y becas privadas no presentan coeficientes estadísticamente significativos.

Se realiza una prueba simple de correlación entre estas variables para examinar su grado de asociación y detectar posibles problemas de multicolinealidad.

\begin{tabular}{|l|c|c|}
\cline { 2 - 3 } \multicolumn{1}{c|}{} & Becas de gobierno & Becas privadas \\
\hline Becas de gobierno & 1 & 1 \\
\hline Becas privadas & $0.5523^{*}$ & \\
\hline
\end{tabular}

*Implica valor estadísticamente significativo al 0.05 .

La correlación es positiva y significativa estadísticamente al 0.05 , por lo que se estiman los valores de tolerancia, factor de inflación de la varianza (VIF, por sus siglas en inglés) y el número condicional para detectar otras situaciones de multicolinealidad.

\begin{tabular}{|lcccc|cc|}
\hline Variable & $\begin{array}{c}\text { Raíz cuadrada } \\
\text { del VIF }\end{array}$ & VIF & Tolerancia & \multicolumn{2}{|c|}{$\begin{array}{c}\text { Eigenvalor } \\
\text { condicional }\end{array}$} & Índice \\
\hline Oportunidades & 1.02 & 1.01 & 0.98 & 1 & 4.69 & 1.00 \\
Remesas & 1.70 & 1.30 & 0.59 & 2 & 0.20 & 4.83 \\
Becas privadas & 1.76 & 1.33 & 0.57 & 3 & 0.07 & 8.01 \\
Becas de gobierno & 1.61 & 1.27 & 0.62 & 4 & 0.03 & 12.68 \\
& & & & 5 & 0.00 & 31.33 \\
\hline
\end{tabular}

Número condicional: 31.3 
Se observa que el valor del VIF para la variable becas privadas es relativamente elevado dentro del conjunto de variables y el valor de la tolerancia para esta variable se aleja considerablemente de la unidad. Por su parte, el cálculo de los eigenvalores para becas del gobierno arroja resultados cercanos a cero, por lo que se tienen indicadores de que existe multicolinealidad. Se excluye la variable becas de gobierno y se estima un segundo modelo.

\section{b) Modelo 2}

$$
\text { ifgt }=a_{0}+\beta_{1} \text { oport }+\beta_{2} \text { becaspriv }+\beta_{3} \text { becasgob }+\beta_{4} \text { remesas }+\mu_{1}
$$

Los resultados de la regresión son los siguientes:

\begin{tabular}{|ccccccc|}
\hline \multicolumn{1}{|c}{$F(3,28)$} & $=$ & 5.3700 & $R^{2}$ & $=$ & 0.3653 & \\
\hline Prob. $>F$ & $=$ & 0.0048 & $\begin{array}{l}R^{2} \\
\text { ajustada }\end{array}$ & $=$ & 0.2972 & \\
\hline $\begin{array}{l}\text { Raíz del error } \\
\text { cuadrático } \\
\text { medio }\end{array}$ & $=$ & 11.2850 & & & & \\
\hline \multicolumn{1}{|c}{ ifgt } & Coeficiente & $\begin{array}{c}\text { Error } \\
\text { estándar }\end{array}$ & $t$ & $P>t$ & $\begin{array}{l}\text { [Intervalo de } \\
\text { confianza de } 95 \%]\end{array}$ \\
\hline Oportunidades & 0.074 & 0.030 & 2.450 & 0.021 & 0.012 & 0.136 \\
\hline Remesas & -0.005 & 0.002 & -1.880 & 0.071 & -0.009 & 0.000 \\
\hline Becas privadas & -0.006 & 0.007 & $-0.92 *$ & 0.365 & -0.020 & 0.008 \\
\hline Constante & 19.851 & 21.598 & 0.920 & 0.366 & -24.390 & 64.092 \\
\hline
\end{tabular}

* No significativo.

Los resultados indican que las variables Oportunidades y remesas son estadísticamente significativas con un nivel del 0.05 , si bien la variable independiente becas privadas no es estadísticamente significativa.

La variable becas privadas se excluye del modelo y se estima la siguiente especificación:

$$
\text { ifgt }=a_{0}+\beta_{1} \text { oport }+\beta_{2} \text { remesas }+\mu_{1}
$$

Los resultados de la regresión son los siguientes: 
78 ECONOMÍA: TEORÍA Y PRÁCTICA • Nueva Época, número 39, julio-diciembre 2013

\begin{tabular}{|lcccccc|}
\hline$F(2,29)$ & $=$ & 7.67 & $R^{2}$ & & 0.35 \\
\hline Prob. > F & $=$ & 0.00 & $R^{2}$ ajustada & & 0.30 \\
\hline $\begin{array}{l}\text { Raíz del error } \\
\text { cuadrático medio }\end{array}$ & $=$ & 11.26 & & & & \\
\hline \multicolumn{1}{|c}{ ifgt } & Coeficiente & $\begin{array}{c}\text { Error } \\
\text { estándar }\end{array}$ & $t$ & $P>t$ & $\begin{array}{c}\text { [Intervalo de confianza } \\
\text { de 95\%] }\end{array}$ \\
\hline Oportunidades & 0.08 & 0.0 & 2.6 & 0.0 & 0.0 & 0.1 \\
\hline Remesas & -0.01 & 0.0 & -3.0 & 0.0 & 0.0 & 0.0 \\
\hline Constante & 11.60 & 19.6 & 0.6 & 0.6 & -28.5 & 51.7 \\
\hline
\end{tabular}

Se observa que tanto las transferencias por remesas como mediante el programa Oportunidades son estadísticamente significativas, sin embargo, se aplica la prueba de White para descomposición de heterocedasticidad de Cameron y Triverdi (homocedasticidad vs. heterocedasticidad) (Greene, 2000).

\begin{tabular}{|lccr|}
\hline$\chi^{2}(5)$ & $=$ & 3.49 & \\
Prob. $>\chi^{2}$ & $=$ & 0.6254 & \\
\hline Fuente & $\chi^{2}$ & $d f$ & 0 \\
Heterocedasticidad & 3.49 & 5 & 0.6254 \\
Asimetría & 2.13 & 2 & 0.3453 \\
Curtosis & 0.85 & 1 & 0.3576 \\
Total & 6.46 & 8 & 0.5959 \\
\hline
\end{tabular}

Los resultados de la prueba de White permiten descartar posibles problemas con la varianza de los residuales, lo que se confirma con la prueba de Bruesch-Pagan.

Ho: Variables con varianza constante. Valores estimados de: ifgt

$$
\begin{array}{l|l}
\chi^{2}(1)=1.00 & \text { Prob. }>\chi^{2}=0.3164
\end{array}
$$

Fuente: Estimación propia con datos de INEGI (2010b).

De esta forma se determina que la especificación de la ecuación (3) es adecuada para estimar el modelo con el método de regresión ponderada geográficamente. Los resultados se discuten a continuación, se consignan en la figura 1 y se incluyen en el cuadro A5. 
Figura 1. Magnitud del impacto en la reducción de la pobreza, según valor de los coeficientes de la variable remesas (regresión ponderada geográficamente)

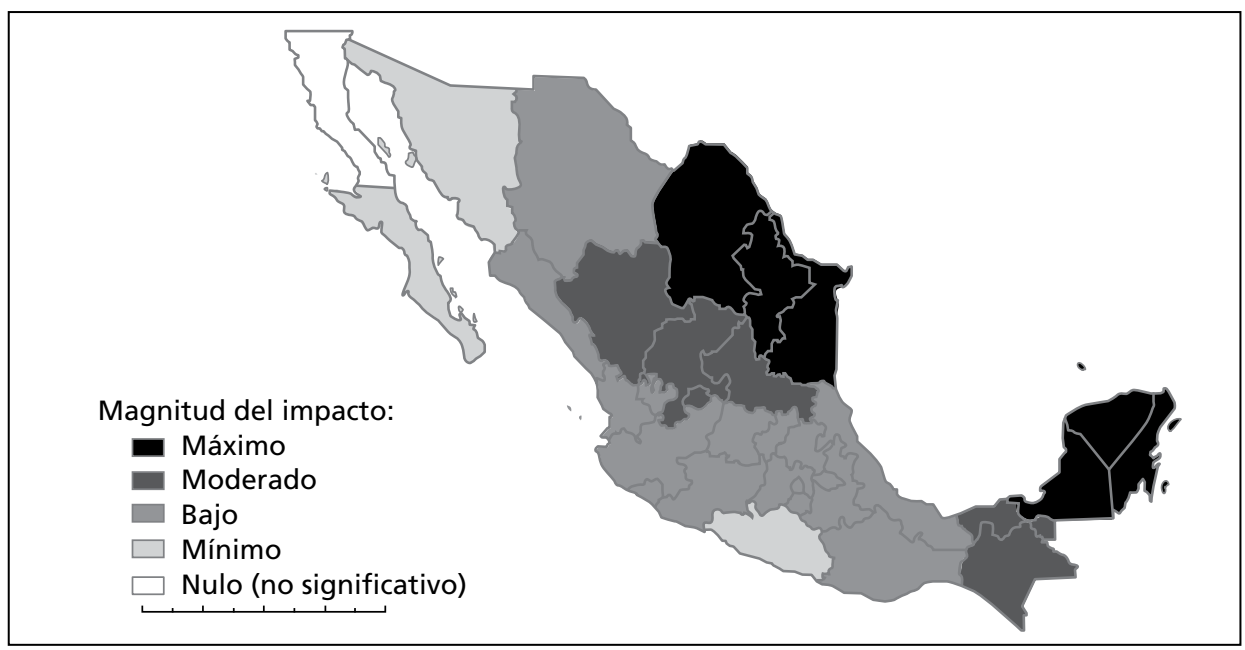

Fuente: Elaboración propia con datos de INEGI (2010a). Cobertura geográfica estatal: Instituto Nacional de Geografía y Estadística (INEGI).

\section{Resultados empíricos}

Los resultados indican que el impacto de las transferencias públicas del programa Oportunidades sobre el indicador de pobreza en el ámbito urbano no es significativo en cuatro estados: Baja California, Sonora, Baja California Sur y Chihuahua. En las restantes 28 entidades la relación observada es positiva, aunque con un impacto marginal. Aparentemente, la implicación de este resultado es que las transferencias del programa Oportunidades en 28 estados no tienen el efecto esperado, sin embargo, es importante señalar que la variable dependiente usada (el índice FGT) es sólo una manera de abordar el complejo tema de la pobreza, por lo que otros esfuerzos de investigación, tal vez con otra metodologías, son necesarios para confirmar esta relación.

En cuanto a la relación entre el indicador de pobreza y el monto estimado de remesas, los resultados indican que estos recursos se asocian con una disminución del grado de pobreza a nivel estatal, es decir, la relación resulta inversa y significativa en 31 entidades del país; la excepción es Baja California. Adicionalmente, se observa que el impacto es mayor en los estados de la región noreste y península de Yucatán, seguidos por la región centro norte, en estados como San Luis Potosí, Zacatecas y Durango. 
La figura 1 muestra la magnitud del impacto a partir de los coeficientes de la regresión ponderada geográficamente (véase el cuadro A5).

\section{CONCLusiones}

El análisis realizado permitió un contraste del impacto de las transferencias en la reducción de la inequidad del ingreso y la pobreza, con una clasificación de recursos en privados y públicos para el periodo 2006-2010. Así mismo, se aprovechó la desagregación de la información para matizar las diferencias en los contextos urbano y rural.

Concretamente, en términos temporales se observó que en 2006, la asignación de transferencias gubernamentales mediante programas sociales como Oportunidades mostró un sesgo favorable a la población rural, subestimando el fenómeno de la pobreza urbana. No obstante, se presentó un crecimiento en términos reales en estos recursos entre 2006 y 2010, con el que si bien se destinó un mayor monto a las localidades rurales, el incremento fue mayor para las urbanas, lo que muestra no sólo el carácter progresivo del esquema de transferencias al beneficiar en mayor medida a los más pobres, sino que también hace evidente la corrección parcial del sesgo que favorecía desproporcionadamente al ámbito rural.

Se verificó una mejoría en la distribución del ingreso atribuible a las transferencias equivalente a $2.7 \%$ del índice de Gini entre 2006 y 2010, principalmente para los deciles de menor ingreso. Sin embargo, este avance se acompañó de un incremento en el número de personas por debajo de la línea de pobreza, que para 2010 se ponderó en 32 millones.

Por otro lado, las estimaciones sobre el carácter en favor de los pobres de los programas de transferencias sociales gubernamentales indicaron que tanto en términos absolutos como relativos, en 2010 se tiene un efecto redistributivo del ingreso en beneficio de los estratos menos favorecidos, con una tasa de crecimiento del ingreso de la población en situación de pobreza (PEGR) de 16\% en comparación con una tasa media de crecimiento del ingreso de sólo 15\%.

El análisis geográfico de los programas de transferencias públicas indica que Oportunidades resultó no significativo en cuatro estados de la República, ubicados en el norte y noreste. En las 28 entidades restantes, el impacto de dicho programa aparentemente no es el esperado en tanto mecanismo para mitigar la pobreza. Sin embargo, el resultado del análisis no es concluyente y debe tomarse con reserva al menos por dos razones: la primera es la naturaleza local de la herramienta econométrica aplicada de regresión ponderada geográficamente y, la 
segunda, la selección del índice FGT como variable para medir la pobreza, ya que es sólo una manera de medir indirectamente este complejo fenómeno.

Respecto al análisis regional del impacto de las transferencias por remesas, se observó que existe una relación inversa respecto al índice FGT, lo que implica que si bien estos recursos contribuyen a reducir la pobreza, el impacto es ínfimo. Esta relación se observó en prácticamente todo el país (exceptuando Baja California), con un mayor impacto en la región noreste y península de Yucatán, y en menor medida en el centro norte del país, en estados como San Luis Potosí, Zacatecas y Durango. Finalmente, es necesario notar que son necesarias futuras investigaciones sobre el tema para complementar estos resultados, dada su importancia para el equilibrio entre crecimiento del ingreso y equidad, así como por la naturaleza compleja del fenómeno de la pobreza en un país como México, que cuenta con flujos importantes de transferencias privadas como las remesas y una gama diversificada de programas sociales.

\section{Anexo}

Cuadro A1. Prueba de hipótesis para diferencia de medias

\begin{tabular}{|c|c|c|c|c|c|c|}
\hline Variable & Obs & Media & $\begin{array}{c}\text { Error } \\
\text { estándar }\end{array}$ & $\begin{array}{l}\text { Desviación } \\
\text { estándar }\end{array}$ & \multicolumn{2}{|c|}{$\begin{array}{c}\text { [Intervalo de confianza } \\
\text { de } 95 \% \text { ] }\end{array}$} \\
\hline $\operatorname{tra} \sim 2010$ & 6107 & 63.397 & 1.8 & 138.5 & 59.9 & 66.9 \\
\hline \multicolumn{3}{|c|}{ Media $=$ media (transferencias_2010) } & $t=2.5364$ & \multicolumn{3}{|c|}{ Grados de libertad $=6106$} \\
\hline \multicolumn{2}{|c|}{ Ho: media $=58.9$} & & & & & \\
\hline \multicolumn{2}{|c|}{ Ha: media $<58.9$} & \multicolumn{2}{|c|}{ Ha: media! $=58.9$} & \multicolumn{2}{|c|}{ Ha: media > 58.9} & \\
\hline \multicolumn{2}{|c|}{$\operatorname{Pr}(T<t)=0.9944$} & \multicolumn{2}{|c|}{$\operatorname{Pr}(T>t)=0.0112$} & \multicolumn{2}{|c|}{$\operatorname{Pr}(T>t)=0.0056$} & \\
\hline
\end{tabular}

Nota: $\mathrm{Ho}=$ homocedasticidad. Ha: heterocedasticidad.

Fuente: Elaboración propia con datos de INEGI (2006; 2010a).

Cuadro A2. Comparativo 2006-2010 de la distribución del ingreso (índice de Gini)

\begin{tabular}{|l|r|r|r|r|r|r|}
\hline \multicolumn{1}{|c|}{ Índice } & Estimación & $\begin{array}{c}\text { Error } \\
\text { estándar }\end{array}$ & \multicolumn{1}{c|}{$t$} & \multicolumn{2}{|c|}{$P>t$} & \multicolumn{2}{|c|}{$\begin{array}{c}\text { [Intervalo de confianza } \\
\text { de 95\%] }\end{array}$} \\
\hline Gini 2006 & 0.51 & 0.00 & 107.01 & 0.00 & 0.50 & 0.52 \\
\hline Gini 2010 & 0.48 & 0.00 & 108.60 & 0.00 & 0.47 & 0.49 \\
\hline Diferencia & -0.03 & 0.01 & -4.13 & 0.00 & -0.04 & -0.01 \\
\hline
\end{tabular}

Nota: Se consideró el ingreso antes de transferencias para la distribución en 2006 y el ingreso con transferencias para la distribución en 2010.

Fuente: Elaboración propia con base en microdatos de INEGI (2006; 2010a). 
82 ECONOMÍA: TEORÍA Y PRÁCTICA • Nueva Época, número 39, julio-diciembre 2013

Cuadro A3. Índice de pobreza SST

\begin{tabular}{|c|c|c|c|c|c|c|}
\hline Periodo & Grupo & Índice SST & $\begin{array}{c}\text { Error } \\
\text { estándar }\end{array}$ & $\begin{array}{c}\text { Límite } \\
\text { inferior }\end{array}$ & $\begin{array}{c}\text { Límite } \\
\text { superior }\end{array}$ & $\begin{array}{c}\text { Línea de } \\
\text { bienestar }\end{array}$ \\
\hline 2006 & Rural & 0.34 & 0.00 & 0.33 & 0.35 & 1070.50 \\
\hline 2010 & Rural & 0.48 & 0.01 & 0.46 & 0.50 & 1330.50 \\
\hline 2006 & Urbano & 0.25 & 0.00 & 0.24 & 0.25 & 1732.50 \\
\hline 2010 & Urbano & 0.30 & 0.00 & 0.29 & 0.31 & 2120.04 \\
\hline
\end{tabular}

Fuente: Elaboración propia con base en microdatos de INEGI (2006; 2010a).

Cuadro A4. Participación en el ingreso por cuartiles

\begin{tabular}{|c|c|c|c|}
\hline Cuartil & 2006 & 2010 & Diferencia \\
\hline 1 & 0.054 & 0.061 & 0.007 \\
\hline 2 & 0.120 & 0.127 & 0.007 \\
\hline 3 & 0.207 & 0.215 & 0.008 \\
\hline 4 & 0.619 & 0.597 & -0.022 \\
\hline
\end{tabular}

Fuente: Elaboración propia con base en microdatos de INEGI (2006).

Gráfica A1. Participación en el ingreso por cuartiles (2006)

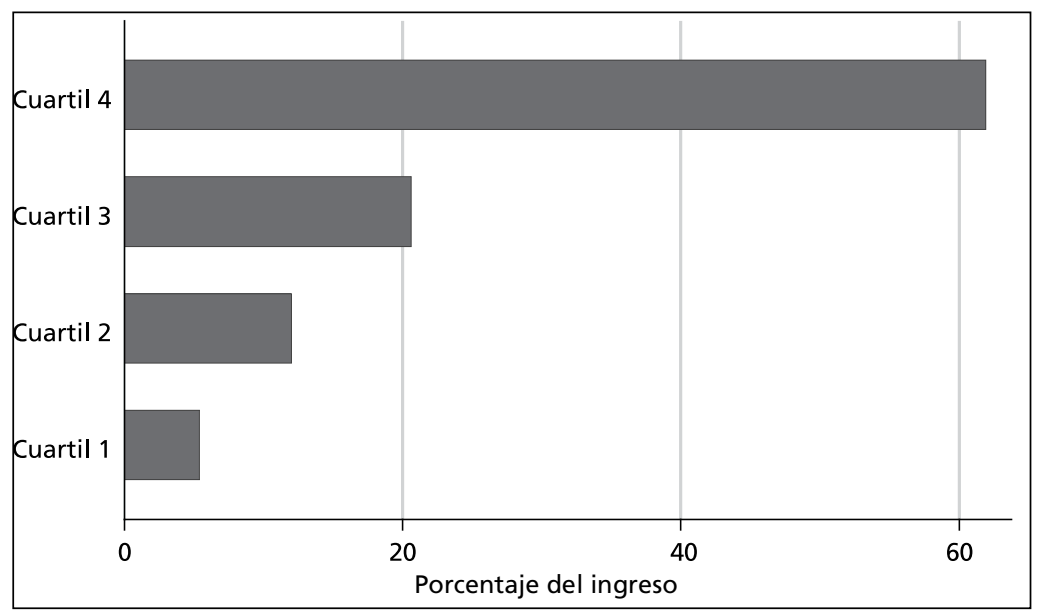

Fuente: Elaboración propia con base en microdatos de INEGI (2006). 
Con propósitos comparativos se presentan los estadísticos de ajuste resultado de la estimación de los modelos 2 y 3.

\begin{tabular}{|c|c|}
\hline \multicolumn{2}{|l|}{ Modelo 2} \\
\hline Estadístico & Valor \\
\hline$\Sigma$ & 11.2 \\
\hline $\mathrm{AlC}_{\mathrm{C}}$ & 253.6 \\
\hline$R^{2}$ & 0.39 \\
\hline$R^{2}$ ajustada & 0.31 \\
\hline Variable dependiente & ifgt \\
\hline Variable independiente & Oportunidades \\
\hline Variable independiente & Remesas \\
\hline Variable independiente & Becas privadas \\
\hline
\end{tabular}

\begin{tabular}{|c|c|}
\hline \multicolumn{2}{|l|}{ Modelo 3} \\
\hline Estadístico & Valor \\
\hline$\Sigma$ & 10.58 \\
\hline $\mathrm{AlC}_{\mathrm{C}}$ & 250.09 \\
\hline$R^{2}$ & 0.48 \\
\hline$R^{2}$ ajustada & 0.38 \\
\hline Variable dependiente & ifgt \\
\hline Variable independiente & Oportunidades \\
\hline Variable independiente & Remesas \\
\hline
\end{tabular}

Fuente: Estimación propia con base en datos de INEGI (2010b).

Se observa que el modelo 3 presenta un mejor ajuste con una $R^{2}$ de 0.48 y con un valor menor que en el modelo 2 para el criterio de información de Akaike corregido (AICC) para estimaciones geográficamente ponderadas de 250, lo cual también representa un mejor ajuste. Asimismo, el modelo 3 también tiene una menor desviación estándar de los residuales, lo que refuerza su selección como modelo de mejor ajuste en comparación con el modelo 2.

\section{Cuadro A5. Resultados de la estimación local para regresión ponderada geográficamente (modelo 3) (continúa)}

\begin{tabular}{|l|c|c|c|c|c|c|c|c|c|}
\hline \multicolumn{1}{|c|}{ Entidad } & LocalR2 & Intercept & C1_opor & C2_rem & t_c1 & t_c2 & $\begin{array}{c}\text { StdErrC1 } \\
\text { E }\end{array}$ & $\begin{array}{c}\text { StdErrC2 } \\
\text { E }\end{array}$ & $\begin{array}{c}\text { Shape_A } \\
\text { km2 }\end{array}$ \\
\hline Aguascalientes & 0.349 & 10.1 & 0.07802912547 & -0.00539871464 & 2.7 & -2.6 & 0.03 & 0.00 & 5559.73 \\
\hline Baja California & 0.284 & 34.4 & 0.02364097332 & -0.00360580028 & 0.6 & -1.6 & 0.04 & 0.00 & 73565.74 \\
\hline $\begin{array}{l}\text { Baja California } \\
\text { Sur }\end{array}$ & 0.304 & 27.7 & 0.04140409645 & -0.00469500522 & 1.3 & -2.4 & 0.03 & 0.00 & 73964.04 \\
\hline Campeche & 0.342 & -0.7 & 0.10163283967 & -0.00585725747 & 3.1 & -2.2 & 0.03 & 0.00 & 51139.30 \\
\hline Coahuila & 0.381 & 17.6 & 0.06597172879 & -0.00572717306 & 2.3 & -2.8 & 0.03 & 0.00 & 150670.31 \\
\hline Colima & 0.329 & 7.4 & 0.08105504843 & -0.00510072379 & 2.8 & -2.4 & 0.03 & 0.00 & 5752.31 \\
\hline Chiapas & 0.339 & -3.1 & 0.10422930891 & -0.00545518867 & 3.1 & -2.0 & 0.03 & 0.00 & 73464.62 \\
\hline
\end{tabular}


84 ECONOMÍA: TEORÍA Y PRÁCTICA • Nueva Época, número 39, julio-diciembre 2013

\section{Cuadro A5. Resultados de la estimación local para regresión ponderada geográficamente (modelo 3) (concluye)}

\begin{tabular}{|c|c|c|c|c|c|c|c|c|c|}
\hline Entidad & LocalR2 & Intercept & C1_opor & C2_rem & t_c1 & t_c2 & \begin{tabular}{|c|} 
StdErrC1 \\
E
\end{tabular} & $\begin{array}{c}\text { StdErrC2 } \\
\text { E }\end{array}$ & $\begin{array}{c}\text { Shape_A } \\
\text { km2 }\end{array}$ \\
\hline Chihuahua & 0.356 & 24.1 & 0.05146980682 & -0.00527879890 & 1.7 & -2.8 & 0.03 & 0.00 & 246991.21 \\
\hline $\begin{array}{l}\text { Distrito } \\
\text { Federal }\end{array}$ & 0.35 & 2.8 & 0.09091035541 & -0.00516646034 & 3.0 & -2.2 & 0.03 & 0.00 & 1487.49 \\
\hline Durango & 0.349 & 17.5 & 0.06448492055 & -0.00548429926 & 2.3 & -2.8 & 0.03 & 0.00 & 122030.63 \\
\hline Guanajuato & 0.35 & 6.9 & 0.08364657839 & -0.00531056025 & 2.8 & -2.4 & 0.03 & 0.00 & 30336.15 \\
\hline Guerrero & 0.343 & 0.9 & 0.09317038202 & -0.00493220890 & 3.0 & -2.1 & 0.03 & 0.00 & 63608.95 \\
\hline Hidalgo & 0.356 & 4.4 & 0.08865093479 & -0.00532143866 & 2.9 & -2.3 & 0.03 & 0.00 & 20653.08 \\
\hline Jalisco & 0.336 & 9.2 & 0.07862977268 & -0.00524935100 & 2.7 & -2.5 & 0.03 & 0.00 & 77952.90 \\
\hline $\begin{array}{l}\text { Estado de } \\
\text { México }\end{array}$ & 0.349 & 3.3 & 0.08986071752 & -0.00515905274 & 3.0 & -2.3 & 0.03 & 0.00 & 22227.39 \\
\hline Michoacán & 0.34 & 5.0 & 0.08591663039 & -0.00509983942 & 2.9 & -2.3 & 0.03 & 0.00 & 58300.20 \\
\hline Morelos & 0.349 & 1.9 & 0.09219881279 & -0.00510169412 & 3.0 & -2.2 & 0.03 & 0.00 & 4861.89 \\
\hline Nayarit & 0.334 & 12.7 & 0.07241734697 & -0.00534266000 & 2.5 & -2.7 & 0.03 & 0.00 & 27771.26 \\
\hline Nuevo León & 0.382 & 13.1 & 0.07468306740 & -0.00576588198 & 2.6 & -2.7 & 0.03 & 0.00 & 63615.16 \\
\hline Oaxaca & 0.347 & -1.9 & 0.09958283099 & -0.00504562083 & 3.1 & -2.0 & 0.03 & 0.00 & 93707.15 \\
\hline Puebla & 0.352 & 1.6 & 0.09347107093 & -0.00518798135 & 3.0 & -2.2 & 0.03 & 0.00 & 34119.27 \\
\hline Querétaro & 0.355 & 5.8 & 0.08605933682 & -0.00533325475 & 2.9 & -2.4 & 0.03 & 0.00 & 11603.60 \\
\hline Quintana Roo & 0.333 & -0.1 & 0.10184280385 & -0.00619448301 & 3.0 & -2.2 & 0.03 & 0.00 & 39146.97 \\
\hline San Luis Potosí & 0.361 & 9.0 & 0.08087316429 & -0.00550120596 & 2.8 & -2.5 & 0.03 & 0.00 & 60462.76 \\
\hline Sinaloa & 0.332 & 20.9 & 0.05717047211 & -0.00530259371 & 2.0 & -2.8 & 0.03 & 0.00 & 56801.37 \\
\hline Sonora & 0.32 & 29.4 & 0.03775743866 & -0.00453531824 & 1.1 & -2.2 & 0.03 & 0.00 & 180936.70 \\
\hline Tabasco & 0.345 & -1.5 & 0.10158328297 & -0.00554072738 & 3.1 & -2.1 & 0.03 & 0.00 & 24701.82 \\
\hline Tamaulipas & 0.379 & 10.0 & 0.08035618416 & -0.00572627114 & 2.7 & -2.6 & 0.03 & 0.00 & 79404.09 \\
\hline Tlaxcala & 0.354 & 2.3 & 0.09214688761 & -0.00522632369 & 3.0 & -2.2 & 0.03 & 0.00 & 3981.92 \\
\hline Veracruz & 0.356 & 1.3 & 0.09480322469 & -0.00532379619 & 3.0 & -2.2 & 0.03 & 0.00 & 71470.07 \\
\hline Yucatán & 0.347 & 1.2 & 0.09924674219 & -0.00613041164 & 3.0 & -2.3 & 0.03 & 0.00 & 37425.48 \\
\hline Zacatecas & 0.354 & 12.4 & 0.07416629466 & -0.00549734375 & 2.6 & -2.7 & 0.03 & 0.00 & 74502.48 \\
\hline
\end{tabular}

Nota: LocalR2 = indicador de bondad de ajuste para cada estado; $C 1 \_o p o r=$ coeficiente estimado para variable Oportunidades; $C 2$ _rem = coeficiente estimado para variable remesas; $t \_c 1$ = estadístico $t$ para variable Oportunidades; t_c2 = estadístico t para variable remesas; StdErrC1_E = error estándar de la estimación en variable Oportunidades; StdErrC2_E = error estándar de la estimación en variable remesas; Shape_A_km2 = área geográfica de cada polígono estatal en kilómetros cuadrados. 


\section{REFERENCIAS BIBLIOGRÁFICAS}

Arzate, Jorge (2010), "Pensar un Estado de bienestar para México: ciudadanía, institucionalidad y economía para el bienestar", en Enrique Valencia Lomelí (coord.), Perspectivas del universalismo en México, Guadalajara, México, ITEso/Fundación Konrad Adenauer, pp. 63-76.

Atkinson, Anthony (1997), "Bringing Income Distribution in from the Cold", The Economic Journal, vol. 107, núm. 441, pp. 297-321.

Banco Mundial (2005), Keeping the Promises of Social Security in the Americas, Washington, DC, Stanford University Press.

Bibi, Sami, y Duclos, Jean-Yves (2005), "Decomposing poverty changes into vertical and horizontal components". Bulletin of Economic Research, vol. 57, núm. 2, pp. 205-215.

Boltvinik, Julio, y Damián, Araceli (2001), “La pobreza ignorada. Evolución y características", Papeles de Población, julio-septiembre, núm. 29, pp. 21-53.

Bourguignon, François, y Spadaro, Amadeo (2006), "Microsimulation as a tool for Evaluating Redistribution Policies", Journal of Economic Inequality, vol. 4, núm. 1, pp. 77-106.

Сво (2011), "The Budget and Economic Outlook: Fiscal Years 2010 to 2022", en Congressional Budget Office. Consultado el 15 de junio de 2012 en: http://www. cbo.gov/ftpdocs/126xx/doc12699/01-31-2012_Outlook.pdf.

CONeval (s.f.a), Medición de la pobreza en los municipios de México, 2010. Nota Tecni$c a$. (PDF), s.l., s.e. Disponible en: http://www.coneval.gob.mx/Informes/Pobreza /Pobreza_municipal/Nota_tecnica/6.1Nota_tecnica.pdf. Consultado el $15 \mathrm{de}$ junio de 2012.

_ (s.f.b), "Medición de la pobreza, líneas de bienestar y canasta básica", en CONEVAL. Consultado el 15 de junio de 2012 en: http://www.coneval.gob.mx/ Medicion/Paginas/Lineas-de-bienestar-y-canasta-basica.aspx.

Datt, Gaurav, y Ravallion, Martin (1992), "Growth and redistribution components of changes in poverty measures. A decomposition with applications to Brazil and India in the 1980s". Journal of Development Economics, vol. 38, núm. 2, pp. 275-295.

Diario Oficial de la Federación (2007, 31 de mayo) "Plan Nacional de Desarrollo 20072012". Disponible en: http://www.diputados.gob.mx/LeyesBiblio/compila/pnd/ PND_2007-2012_31may07.doc. Consultado el 20 de junio de 2012.

- (2010, 31 de diciembre), "Acuerdo por el que se modifican las reglas de operación del Programa de Apoyo Alimentario, para el ejercicio fiscal 2011”, México. 
Duclos, Jean-Yves (2009). What is "Pro-Poor"? Social Choice Welfare, vol. 32, núm. 1, pp.37-58.

Duclos, Jean-Yves, y Verdier-Chouchane, Audrey (2010), “Analyzing Pro-Poor Growth In Southern Africa: Lessons From Mauritius And South Africa”, working paper núm. 115, African Development Bank.

Foster, James; Greer, Joel, y Thorbecke, Erik (1984), “A class of decomposable poverty measures", Econometrica, vol. 52, núm. 3, pp.761-766.

Förster, Michael, y d'Ercole, Marco (2005), "Income Distribution and Poverty in OECD Countries in the Second Half of the 1990s", OECD Social, Employment and Migration Working Papers núm. 22, París.

Fotheringham, Stewart; Brunsdon, Chris, y Charlton, Martin (2002), Geographically Weighted Regression: the analysis of spatially varying relationships, New York, John Wiley \& Sons,

Gobierno Federal (2007), Politica social Vivir Mejor, Mexico.

Greene, William (2000), Econometric Analysis, 4a ${ }^{\text {. }}$ edicion, Upper Saddle River, New Jersey, Prentic-Hall.

Huesca, Luis (2010). “Análisis del programa Oportunidades en México: Impacto en la distribución de una aplicación universal por tipos de pobreza", en: Enrique Valencia Lomelí (coord.), Perspectivas del universalismo en México, Guadalajara, México, ITESo/Fundación Konrad Adenauer, pp. 195-207.

INEGI (2006), "Encuesta Nacional de Ingresos y Gastos de los Hogares 2006”, en Instituto Nacional de Estadística y Geografia. Consultado el 16 de julio de 2012 en: http://www.inegi.org.mx/est/contenidos/proyectos/encuestas/hogares/regulares/ enigh/enigh2006/default.aspx.

(2010a), "Encuesta Nacional de Ingresos y Gastos de los Hogares 2010”, en Instituto Nacional de Estadística y Geografía. Consultado el 16 de julio de 2012 en: http://www.inegi.org.mx/est/contenidos/Proyectos/Encuestas/Hogares /regulares/Enigh/Enigh2010/tradicional/.

- (2010b), "México. Módulo de Condiciones Socioeconómicas de la ENIGH 2010", en Instituto Nacional de Estadística y Geografía. Consultado el 16 de julio de 2012 en: http://www3.inegi.org.mx/rnm/index.php/catalog/152.

Kakwani, Nanak, y Pernia, Ernesto (2000). "What is pro-poor growth?", Asian Development Review, vol. 16, núm. 2, pp. 1-22.

Kakwani, Nanak; Khandker, Shahid, y Son, Hyun (2003), "Poverty equivalent growth rate: with applications to Korea and Thailand", reporte técnico a la UN ECA Expert Group Meeting, en Kampala, Uganda, 23 y 24 de junio, s.1., Economic Commission for Africa/Economic Policy Research Center. 
- (2004), "Pro-poor growth: concepts and measurement with country case studies", working paper núm. 1, United Nations Development Programme.

OECD (2011), "Society at a Glance 2011 - OECD Social Indicators", en $O E C D$. Consultado el 11 de julio de 2012 en: http://www.oecd.org/els/soc/societyataglance.htm Rawls, John (1971), A theory of justice, Cambridge, Harvard University Press.

Rubalcava, Rosa María (2010), "La perspectiva territorial del universalismo en México", en Enrique Valencia Lomelí (coord.), Perspectivas del universalismo en México, Guadalajara, México, ITESo/Fundación Konrad Adenauer, pp. 223-229.

Ravallion, Martin, y Chen, Shaohua (2003), "Measuring pro-poor growth". Economics Letters, vol. 78, núm. 1, pp. 93-99.

Scott, John (2004), “La descentralización, el gasto social y la pobreza en México". Gestión y Política Pública, vol. XIII, núm. 3, pp. 785-837.

— (2005), "Seguridad social y desigualdad en México: de la polarización a la universalidad”. Bienestar y Política Social, vol. 1, núm. 1, pp. 59-82.

SEDESOl (2012), Meta evaluación 2007-2012 del Programa 70 y Más. Informe final. (PDF), Tuxtla Gutiérrez, Secretaría de Desarrollo Social. Disponible en: http:// www.2006-2012.sedesol.gob.mx/work/models/SEDESOL/Resource/3158/1/ images/70yMas_METAEVALUACION.pdf. Consultado el 11 de julio de 2012 (s.f.), "Programa 70 y Más". Consultado el 17 de octubre de 2012, en: http:// www.sedesol.gob.mx/en/SEDESOL/programa_70_y_mas.

Walle, Dominique van de (1995), "Incidence and Targeting: An Overview of Implications for Research and Policy", en Dominique van de Walle y Kimberly Nead, Public Spending and the Poor: Theory and Evidence, Baltimore and London, The Johns Hopkins University Press for the World Bank.

Wooldridge, Jeffrey (2000), Introductory Econometrics: A Modern Approach, Mason, $\mathrm{OH}$, South Western College.

Xisco, Oliver; Luca, Piccoli, y Spadaro, Amadeo (2010), “A microsimulation evaluation of efficiency, inequality, and polarization effects of implementing the danish, the french, and the UK Redistribution System in Spain". Review of Income and Wealth, vol. 56, núm. 1, pp. 186-214. 